\title{
Evaluation of 12-DOF Input Devices for Navigation and Manipulation in Virtual Environments
}

\author{
Anke Huckauf, Alexander Speed, André Kunert, Jan Hochstrate, and Bernd Fröhlich \\ Faculty of Media, Bauhaus-University Weimar, Germany \\ \{anke.huckauf, alexander.speed, andre.kunert, jan.hochstrate, \\ bernd.froehlich\} @medien.uni-weimar.de
}

\begin{abstract}
Navigation and manipulation in virtual environments may require up to six degrees of freedom each. Input devices with twelve or more degrees of freedom can avoid explicit changes between navigation and manipulation and may therefore perform well in certain situations. However, usability of already existing 12-DOF devices is still unclear. For evaluating such handheld devices, we developed an extended docking task based on docking tasks designed for examining the usability of 6-DOF devices. In addition to the usually investigated object manipulation, the task requires navigation. We compared docking performances of two 12-DOF devices, the CubicMouse and the YoYo. Additionally, performance with a newly developed 12-DOF input device, the SquareBone, was under study. The SquareBone, a variation of the YoYo idea combined with some potentially beneficial features of the CubicMouse, provides $2 * 6$ elastic DOF which can be controlled simultaneously. The study revealed that the isotonic CubicMouse, although preferred by novice users, was outperformed by the elastic SquareBone and the YoYo. The new SquareBone was shown to bear the potential of becoming superior to the YoYo, possibly because it enables simultaneous control of the $2 * 6 \mathrm{DOF}$.
\end{abstract}

\section{Introduction}

Navigation and object manipulation are central interaction tasks for three-dimensional virtual environments. In most virtual reality (VR) systems these tasks are controlled through a single 6-degree of freedom (DOF) input device. In this case, switching between navigation and manipulation requires a mode change through a menu, button press, or some type of command. This additional operation may not only be timeconsuming, but may also lead to confusion between modes. Input devices with 12 or more DOF could avoid this explicit mode change and may perform better for tasks which require frequent changes between navigation and manipulation.

Input devices can be classified according to the range of motion and their manipulation resistance. Isotonic devices (e.g., the 2-DOF computer mouse) allow forceless movements while measuring travel. Isometric devices are controlled by using force and provide infinite movement resistance. Elastic devices allow slight force-requiring movements. Isometric and elastic devices measure the applied force. The input is mapped onto the movement of objects via position control (i.e., the position of the device is used to control the position of the object) or via rate control 
(i.e., the force applied to the device is used to control the velocity of the object). Zhai [16] showed that the combinations of isotonic sensors and position control, and of elastic or isometric sensors and rate control produce superior performance when compared to other combinations.

Another important factor is the compatibility of integral attributes of a task with simultaneously available DOF of the input device [9]. If a task requires movement in all three spatial directions, the input device should support these translations in multiple axes. Therefore, to make and understand design decisions, one should also consider typical applications for the evaluation. This is especially the case for the multiple-DOF devices presented here which are controlled by both hands.

Hinckley and co-workers [ $[8]$ use a head prop for neurosurgical visualization. Users hold a small rubber sphere or a doll's head with an embedded tracker in one hand. This head prop is used to control the orientation of a head model on the screen. The other hand holds a second prop which can be used to position a cutting plane relative to the head prop. Although using two separate input devices is certainly an alternative, we focus on approaches where both hands manipulate a single device. A variety of systems using two-handed interaction techniques is based on handheld widgets. In [13], for example, users hold a virtual widget in one hand and operate it with the other. In [14], users hold a miniature model of the virtual world in one hand and manipulate objects in the miniature with the other. These systems often employ tracked data gloves in combination with direct manipulation techniques which are difficult to use with large projection systems.

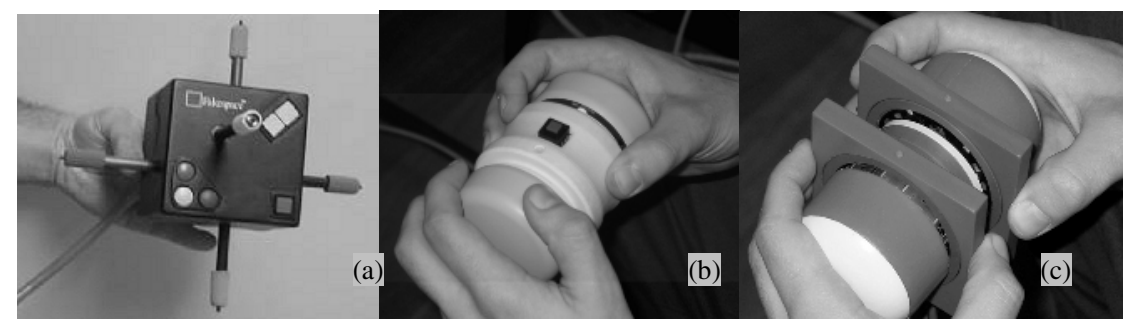

Fig. 1. (a) The CubicMouse is commercially available. The cube-shaped box is held with the non-dominant hand, while the dominant hand manipulates the rods. (b) The YoYo consists of two SpaceMouse sensors attached to a handle in the middle. (c) The SquareBone's handles attached to the contained SpaceMouse sensors can be simultaneously manipulated with the finger tips.

\subsection{The CubicMouse}

The CubicMouse $[\underline{4}, \underline{5}]$ is a handheld interface device consisting of a cube-shaped box with a 6-DOF tracking sensor and three orthogonal rods, each offering 2 DOF (Fig. 1a). It can thus be described as a $6+(3 * 2)$ DOF device, basically a handheld 3-D coordinate system, for which the rods represent the $\mathrm{X}, \mathrm{Y}$, and $\mathrm{Z}$ axis. The rods can be pushed, pulled, and twisted. Pushing or pulling a rod is used for translating an object in the respective direction. Twisting a rod rotates an object around the corresponding axis. The 6-DOF sensor tracks the device movements used for view manipulations. The CubicMouse employs position control for all DOF. It is mostly used for data 
visualization applications, where the box represents a three-dimensional model, and translations and rotations of the box are directly applied to the model. The rods manipulate data probes such as cutting planes. The device allows simultaneous manipulation of up to eight DOF; six DOF offered by the cube plus operation of one of the rods which adds another two DOF.

\subsection{The YoYo}

The YoYo [15] consists of two symmetric elastic 6-DOF SpaceMouse sensors attached to a grip in the middle (Fig. 1b). This $2 * 6$ DOF device is used with rate control techniques. The device is operated with the caps of the SpaceMouse sensors. The left SpaceMouse is typically used for navigation, the right SpaceMouse for object manipulation. Switching between navigation and manipulation requires changing the grip on the device. It is common to embed a 6-DOF tracker in the grip to compensate for rotations of the YoYo against the world coordinate system. The tracker may also be used for isotonic input and position controlled rotation or translation. This mode was not enabled in our study.

\subsection{The SquareBone}

We aimed at examining the usability of the CubicMouse and the YoYo; both, however, differing in various features: Whereas the CubicMouse offers isotonic control, the YoYo provides elastic control for all 12 DOF. Hence, position control is applied for the CubicMouse, and rate control for the YoYo. The CubicMouse is a $6+3 * 2$ DOF device, and the YoYo offers $2 * 6$ DOF. Besides these principal differences, navigation and manipulation can occur simultaneously only with the CubicMouse by moving one rod simultaneously with the cube. The YoYo allows only sequential control due to re-grasping necessary to shift between left and right 6-DOF sensors. Furthermore, due to its cubic shape, the CubicMouse provides a tactile coordinate system which might serve as a reference for navigation, whereas the round YoYo does not provide information about its current orientation.

In order to differentiate between potentially important features for 12-DOF devices, we developed the SquareBone (Fig. 1c). The SquareBone belongs to the YoYo family and consists of $2 * 6$ DOF SpaceMouse sensors as well. The caps of the SpaceMouse sensors are replaced by square handles, providing a distinct tactile coordinate system. As for the YoYo, the handles are manipulated with the fingertips. Contrary to the YoYo, both handles can be operated simultaneously by both hands. The SquareBone has its grip on the outside and rests comfortably in the user's hands.

\section{The Extended Docking Task}

The manipulation of an object in a virtual world is one necessary and main objective for an evaluation task of 12-DOF devices. Several standard tasks have been developed to study the usability of 6-DOF devices under controlled conditions [e.g., 11, 16]. Typical tasks measure docking performance. Docking requires the manipulation of one object (the cursor) which has to be moved to another position or object (the target). To avoid that objects obscure each other, stimuli are usually presented with 
transparent surfaces. In real environments, such occlusions are less problematic since one can easily change the point of view. Hence, inducing a feeling of real world presence should be increased via allowing for changes of the view point, that is, via navigation. Navigation is provided by devices allowing for 12-DOF input. A standard task to study performance within 12 DOF should require to change the view in addition to the manipulation of an object. Our suggested solution is the extended docking task (Fig. 2). In this task, participants have to manipulate a part of a cube (the cursor) to dock it onto a larger static counter-part (the target). The target is positioned so that participants are forced to change their view point for fast and precise docking.

One problem with such an extended docking task is its increased complexity compared to a typical 6-DOF docking task [e.g., 16]. As a consequence, task performance is harder to interpret with respect to device features: Since the task is rather difficult and might require a lot of cognitive processing, completion times can be also due to various task difficulties. In addition, task difficulty may produce a large variability so that even in case of existing differences between various devices or device features, their effects may be masked. Various approaches were included for best possible control of such effects. We used a within-design in which participants worked with several devices. Although this requires careful control of transfer effects, equal task difficulties can be supposed when comparing the devices.
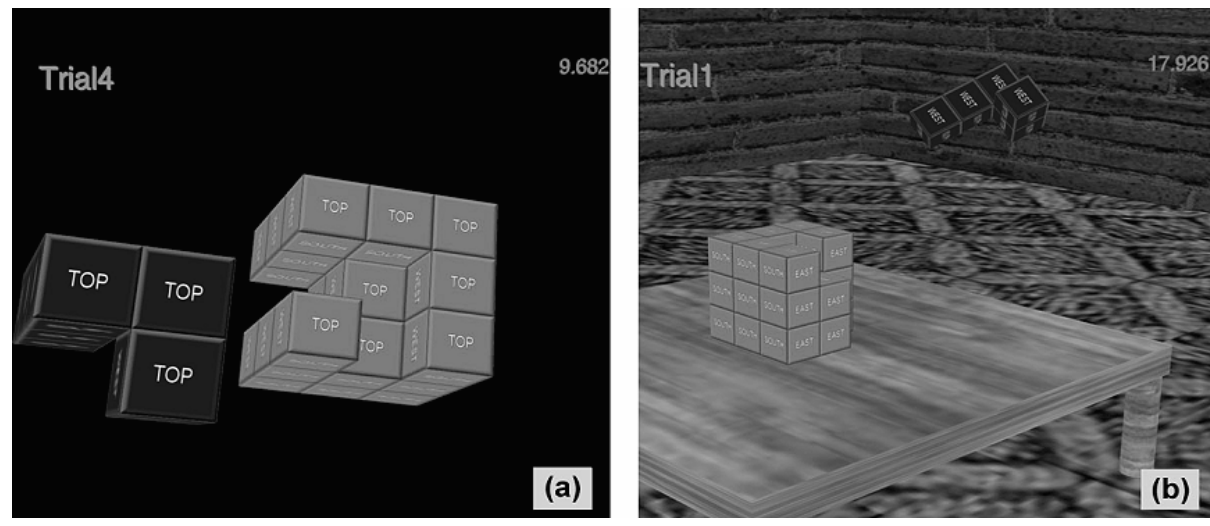

Fig. 2. Stimuli in the extended docking task consist of a larger bright (red) target composed of $3^{3}$ small cubes minus five adjacent small cubes, which represent the dark (blue) cursor. Cursor and target may appear (a) in front of a black background, or (b) inside a virtual room.

In addition, we investigated two possible modes for navigation, an egocentric and an exocentric one. Egocentric mode means that the own point of view is manipulated. That is, pushing the device to the left results in movements of the view to the left. Thus, the world moves to the right (i.e., the device input is interpreted relative to the current view position). In exocentric mode, the world is moved with the device. Hence, moving the device to the left results in a movement of the world to the left (i.e., the device input is interpreted relative to a position of an object in the world). Obviously, the exocentric mode implies that the one point in the world serving as 
centre of all navigation movements is being interpreted as the centre of attention [1]. In fact, this mode reduces the potential navigation trajectories. However, the CubicMouse has been designed to be used in an exocentric mode, and egocentric navigation turned out to be hard to understand with this device. This can be attributed to the isotonic navigation in that the cube of the CubicMouse serves as a prop for the referenced object.

To gain information of whether there is a similar preference for devices employing elastic navigation, we collected some first preliminary data with the SquareBone. Besides the kinds of navigation control (exocentric, egocentric), we varied the structure of the virtual world. Exocentric navigation (i.e., moving the world) might be supported by displaying a certain isolated object, whereas egocentric navigation (i.e., moving one's own view) might benefit from a virtual world around the target, which may provide a reference frame. Preliminary data of 16 novice users showed that exocentric navigation was about $30 \%$ faster when presenting the target in front of a black background compared to being presented inside a virtual room. For egocentric navigation, the opposite effect was observed, although the effect was much smaller (about 2\%). In addition in more than $20 \%$ of trials, neither the cursor nor the target have been visible on the screen for more than $10 \mathrm{sec}$ suggesting that the user got lost within the virtual world. As already the TCTs, these aberrant movements were systematically affected by the interaction of the navigation mode and the background: About $35 \%$ occurred in the egocentric, and $65 \%$ in the exocentric mode of navigation. From all aberrations in the egocentric mode, $75 \%$ occurred with the black background, whereas from all aberrations in the exocentric mode, more than $90 \%$ occurred within the room.

This finding led to two consequences: First, a bounding box around the scene was implemented in order to avoid aberrant movements. Second, exocentric navigation was always presented with a black surround, whereas egocentric navigation was performed within a room. Another important factor is the compatibility between integral and separate parts of task and the simultaneously available DOF of a device [9]. For the task at issue, navigation and object manipulation can be considered to be separate parts which are therefore to be controlled by separate subsets of the totally available DOF. The less frequent navigation was assigned to the subdominant hand known to perform movements of higher amplitude and lower frequency than the dominant one [7].

\section{Experiment 1}

Experiment 1 aimed at establishing the usability of the isotonic CubicMouse, the elastic YoYo, and the elastic SquareBone with our new task. Training functions were assessed over nine sessions within five weeks. In addition, subjective ratings of various device features were collected. All three devices were tested with the exocentric navigation mode. Since egocentric navigation typically implemented using rate control techniques does not work well with isotonic devices [16], egocentric navigation was studied with the Yoyo and the SquareBone only. 


\subsection{Methods}

The stimuli consisted of a large cube composed of $3^{3}$ small cubes (Fig. 2). Five small cubes were removed from the large cube and formed the cursor. The remaining cubes served as target. The target's cubes were red, whereas the cursor's cubes were blue and changed to red individually once they were positioned within the docking tolerance. The change in color served as feedback for the participants. The target was always placed with its missing part on the back-side so that it was almost impossible to dock the cursor without manipulating the view. There were five different starting positions of the cursor each with a certain orientation of the view of approximately equal difficulty. Translation and rotation values of the device were recorded at $20 \mathrm{~Hz}$.

Eight students already experienced with various input devices for threedimensional virtual environments volunteered to participate in the experiment. The order of devices was balanced across participants. All three devices were used by half of the participants with the exocentric mode of navigation (10 trials per session), and the other half used the SquareBone and the YoYo with the egocentric mode (15 trials per session). Participants were assigned to each group according to their performance in a mental rotation test [ $[\underline{6}$ to assure for comparable task difficulty in both groups. For the exocentric mode, target and cursor were presented before a black surround. Egocentric navigation was performed within the room environment. Participants performed the task on nine days with a two or three days break in between.

Participants were seated in front of a 22" monitor and performed the task in monoscopic display mode. In the first session, the task and the device were explained by performing two training trials. The participants' right hand was measured, and preliminary experience with computers and various input devices as well as handcrafting skills were assessed. After the first, the fourth, and the eighth session, the participant filled out written questionnaires about the devices asking about various features, each on a four-point scale. The experiment ended with a last questionnaire consisting of three questions designed to compare the tested devices. One experimental session took about 45 minutes.

\subsection{Results and Discussion}

Device performance and learning. Data was averaged across sessions and entered into a 9 (session: 1 to 9) * 2 (device: YoYo, SquareBone)-ANOVA with repeated measures and the between factor navigation mode (exocentric, egocentric). Only for the exocentric group, an additional 9 (session) *3 (device: YoYo, SquareBone, CubicMouse) was performed. Effects not reported failed to reach significance.

Mean TCTs for all participants using the YoYo and the SquareBone decreased from session 1 (27.46 sec, se=3.06) to session 9 (15.09 sec, se=.53; $\mathrm{F}_{(8,48)}=18.10$; p $<.001$, see Fig. 3). The SquareBone tended to produce better performance than the YoYo, but only during some sessions within the exocentric mode of navigation (interaction between session, device, and mode: $\mathrm{F}_{(8,48)}=1.90 ; \mathrm{p}<.10$ ). The analysis only for the group performing the task with all three devices revealed that mean TCTs for the CubicMouse were significantly larger than for the other devices $\left(\mathrm{F}_{(82,6)}=266.44\right.$; $\mathrm{p}<.001)$. The interaction between session and device was of significance $\left(\mathrm{F}_{(16,48)}=2.95 ; \mathrm{p}<.05\right)$. 


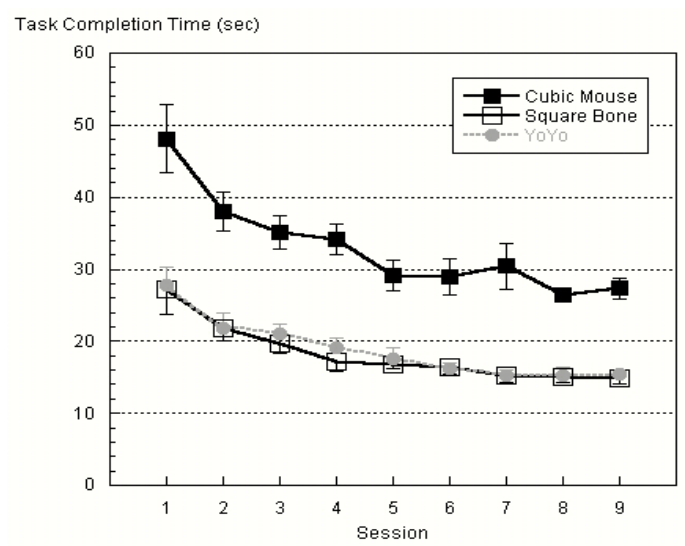

Fig. 3. Mean task completion times and standard errors as a function of session separately for the three devices in Experiment 1

Table 1. Mean estimated intercept and exponent values of the individually fitted power functions as well as the determination coefficient $\mathrm{R}^{2}$. Standard errors are given in brackets.

\begin{tabular}{ccccc}
$\begin{array}{c}\text { Navigation } \\
\text { mode }\end{array}$ & Device & $\begin{array}{c}\text { Intercept } \\
{[\mathrm{sec}]}\end{array}$ & $\begin{array}{c}\text { Exponent } \\
{[\mathrm{sec}]}\end{array}$ & $\mathrm{R}^{2}$ \\
\hline egocentric & SquareBone & $26.98(5.51)$ & $-.22(.09)$ & $.81(.14)$ \\
& YoYo & $29.11(4.73)$ & $-.27(.08)$ & $.88(.11)$ \\
\hline & SquareBone & $25.13(4.84)$ & $-.27(.07)$ & $.69(.12)$ \\
exocentric & YoYo & $26.88(4.10)$ & $-.26(.07)$ & $.64(.09)$ \\
& CubicMouse & $46.87(4.06)$ & $-.26(.02)$ & $.84(.05)$
\end{tabular}

To further examine the question whether the learning progress differs between devices, power functions were fitted. As the intersection values confirmed (see Tab. 1 ), the CubicMouse performed worse than the other devices. There was a tendency of the SquareBone to produce better performance than the YoYo. However, exponents did not differ showing that performances declined at about the same rate for all devices.

Learning should not only result in faster docking performance, but also in a reduction of variability. Standard deviations for all participants using the YoYo and the SquareBone were reduced from $9.17 \mathrm{sec}$ in session 1 to $3.37 \mathrm{sec}$ in session 9 $\left(F_{(8,48)}=10.31, p<.01\right.$, see Fig. 3). The decrease was marginally more pronounced in the egocentric mode than in the exocentric mode $\left(\mathrm{F}_{(8,48)}=1.95 ; \mathrm{p}<.10\right)$ in that it decreased from $9.89 \mathrm{sec}$ to $3.54 \mathrm{sec}$ in the egocentric, and from $8.45 \mathrm{sec}$ to $3.20 \mathrm{sec}$ in the exocentric group. For the exocentric group, variability was shown to depend on sessions $\left(\mathrm{F}_{(8,24)}=5.56, \mathrm{p}<.01\right)$, on the device $\left(\mathrm{F}_{(2,6)}=14.25, \mathrm{p}<.01\right)$, and on their interaction $\left(\mathrm{F}_{(16,48)}=2.36 ; \mathrm{p}<.05\right)$ : Whereas variability for the SquareBone decreased from $8.60 \mathrm{sec}$ to $2.66 \mathrm{sec}$, for the YoYo the decline was less steep (from $8.30 \mathrm{sec}$ to $3.74 \mathrm{sec}$ ). For the CubicMouse, variability decreased steeper on a higher level (from $15.75 \mathrm{sec}$ to $6.62 \mathrm{sec}$ ). 
Simultaneous control. One central difference between the devices is the degree to which simultaneous control of navigation and manipulation is possible. The portions of time during which both, cursor and view, were manipulated at the same time, were compared. Over all participants, this portion was $1.78 \%(\mathrm{se}=.49)$ for the SquareBone, and $0.45 \%$ (se=.16) for the YoYo - a marginally significant difference $\left(F_{(1,6)}=3.97\right.$, $\mathrm{p}<.10)$. In addition, the exocentric group produced more simultaneous control $(6.93 \%)$ than the egocentric group $\left(1.05 \% ; \mathrm{F}_{(1,6)}=5.33 ; \mathrm{p}=.06\right)$. Moreover, a significant interaction between session and device $\left(\mathrm{F}_{(16,48)}=1.90, \mathrm{p}<.05\right)$ revealed that whereas simultaneous control with the YoYo slightly decreased over sessions (1.52\%:1.29\% in session 1:9), its portion increased for both other devices (SquareBone: 1.37\%, $2.14 \%$, CubicMouse: $9.42 \%, 16.07 \%$ ). This suggests that simultaneous control is learned over sessions. One might wonder about the simultaneous control observed for the YoYo: This is due to two participants with the smallest hands, who also stated that the YoYo was too large accidentally causing involuntary movements with the grasping hand.

However, it is still unclear whether simultaneous control is of general advantage. If simultaneous control can produce an advantage, then TCTs should become shorter with increasing simultaneous control; that is, a negative correlation between TCTs and the fraction of simultaneous control is to be expected. For the trials of session 9, TCTs and portion of simultaneous control significantly correlated for the SquareBone with $\mathrm{r}=-.49(\mathrm{p}<.001)$, whereas for the YoYo, there was with $\mathrm{r}=.10$ no significant correlation $(\mathrm{p}=.30)$. This confirms the interpretation that the amount of simultaneous control with the YoYo was mainly due to involuntary movements. It further supports the assumption that simultaneous control can produce advantages at least for experienced users, and therefore can be assumed to provide one important feature of a 12-DOF input device. But, it should be noted that the correlation for the SquareBone traces back mainly to two participants. For the CubicMouse, however, TCTs and the fraction of simultaneous control of cursor and view did not correlate $(r=-.13, p=.42)$. This can be attributed to the users' reports of problems in stabilizing the view with the CubicMouse: Since changing ones own position leads to changes of the view with the CubicMouse, one has to control not only arms and hands, but also feet and body postures. That is, the way of how simultaneous control is enabled severely affects performance. For precise manipulation of an object with the CubicMouse, it might be effective to temporarily disable navigation by clutching.

Times of inactivity and subjective ratings. For all participants using the YoYo and the SquareBone, the fraction of times in which no movement was visible for any DOF was in mean $13.62 \%$ ( $\mathrm{se}=.95$ ) and decreased with increasing training from $16.82 \%$ in session 1 to $12.18 \%$ in session $9\left(\mathrm{~F}_{(8,48)}=4.09 ; \mathrm{p}<.01\right)$. Only for the exocentric group, except the effect of session, also an effect of device was obtained $\left(\mathrm{F}_{(2,6)}=54.15\right.$; $\mathrm{p}<.001$ ) showing that the relative amount of time in which no signal was transferred to any DOF was for the CubicMouse with $30.94 \%$ twice as large as for the two other devices. This difference, which is even larger if one takes the absolute (longer) docking times with the CubicMouse into consideration, is very likely to reflect times of re-grasping, which are much longer for the CubicMouse where each rod has to be accessed and moved separately. Correlations between TCTs and the fraction of inactivity did not reach significance for any device (SquareBone: $r=-.08$. YoYo: 
$\mathrm{r}=-.09$, CubicMouse: $\mathrm{r}=.04$ ). This suggests that times of inactivity seem in part due to cognitive processing. This interpretation is supported by the fact that the fraction of time of inactivity correlated between devices $(\mathrm{r}=.43 ; \mathrm{p}<.01)$.

Subjective ratings of usability (on a four-point scale ranging from $1=$ very poor to $4=$ very good) showed that ratings increased with increasing training from $2.7(\mathrm{se}=.10)$ to $3.4(\mathrm{se}=.08)$ for all participants and the YoYo and the SquareBone. Only for the exocentric group, there was an additional effect of device in that the CubicMouse received worse usability ratings $(1.8, \mathrm{se}=.09)$ than the YoYo $(3.0, \mathrm{se}=.23)$ and the SquareBone (3.1, se=.21).

Taken together, the results show that docking performance with the SquareBone and the YoYo is better than with the CubicMouse. The data tend to demonstrate small advantages for the SquareBone over the YoYo in mean docking duration, in variability during performing the task, and in adequate size. Nevertheless, reducing the size of the YoYo might be an option to overcome these slight advantages of the SquareBone.

\section{Experiment 2}

The input devices have been developed for being used with large projections screens and potentially stereoscopic virtual environments. Since the data in Experiment 1 was collected under monoscopic viewing conditions using a monitor, the participants of Experiment 2 performed the task again using a monitor as well as a large projection screen $\left(4 * 3 \mathrm{~m}^{2}\right)$, both under monoscopic as well as stereoscopic viewing conditions. For the monitor, we used active stereo and shutter glasses. For the large screen, we used passive stereo with linear polarization.

\subsection{Results and Discussion}

As can be seen in Fig. 4, performance did not differ between conditions (all $\mathrm{F}<1$ ). This indicates that the results of our long term Experiment 1 can be transferred to large screens and stereo conditions. Over all participants using the SquareBone and the YoYo, there was one significant interaction between the device, the mode of view, and the output device $\left(\mathrm{F}_{(1,6)}=42.36, \mathrm{p}<.001\right)$. This interaction is due to the relatively large difference between the YoYo and the SquareBone when the task is performed in stereoscopic mode at the projection screen. The group working with the exocentric mode of navigation produced the interaction between device, and the output device $\left(\mathrm{F}_{(2,6)}=7.28, \mathrm{p}<.05\right)$ due to the fact that performance with all devices except the SquareBone was worst under stereoscopic viewing conditions at the large projection screen. In addition, a main effect of device $\left(\mathrm{F}_{(2,6)}=119.64, \mathrm{p}<.001\right)$ showing that performance with the CubicMouse of $26.60 \mathrm{sec}$ ( $\mathrm{se}=.42$ ) was again worse than with the other devices (SquareBone: $12.75 \mathrm{sec}$, se=.95; YoYo: $14.18 \mathrm{sec}$, se=1.13).

In summary, the effects of devices already observed in Experiment 1 were replicated in Experiment 2 in that performance with the CubicMouse was worse than performance for the SquareBone and the YoYo. Again, slight advantages for the SquareBone relative to the YoYo were obvious. Moreover, the data gave rise to the assumption that the SquareBone might produce even more advantages under more 
typical stereoscopic viewing conditions using a large projection screen. Of course, it cannot be ruled out that participants trained in stereo on the projection screen produce better performance under these conditions. But, we can at least proceed on the assumption that the fundamental differences between performances for the devices under study hold for immersive three-dimensional environments as well.

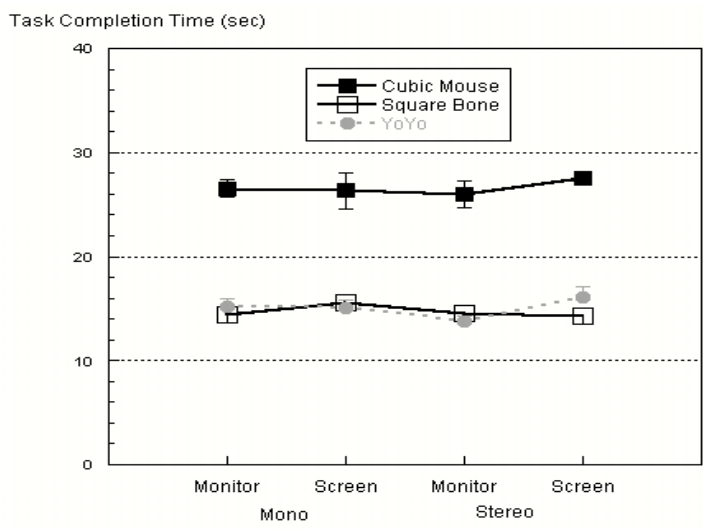

Fig. 4. Mean docking performance for monoscopic and stereoscopic mode of view and monitor and large projection screen for all three devices

\section{Experiment 3}

Experiments 1 and 2 have shown that the isotonic CubicMouse is clearly outperformed by the elastic devices. However, for 6-DOF devices Zhai [16] reported comparable, for novice users even superior performances for isotonic relative to elastic or isometric control. Therefore, in Experiment 3, novice users performed the extended docking task with the SquareBone and the CubicMouse. Eight users performed ten trials with exocentric navigation; four of them first operated the CubicMouse, four the SquareBone.

\subsection{Results and Discussion}

Mean TCT with $67.90 \mathrm{sec}$ as well as variability with a standard error of $2.49 \mathrm{sec}$ were quite high over all 10 trials. This can be attributed to the high task difficulty, especially for the completely inexperienced users.

To study short term learning effects, the first and the last (i.e., tenth) trial were statistically compared. Both devices produced comparable performances (CubicMouse: 74.33 sec, se=8.05; SquareBone: 75.92 sec, se=8.44; $\mathrm{F}<1$ ). Performance in the last trial was better than in the first one $\left(F_{(1,6)}=9.82, p<.05\right.$, Fig. 5) revealing training effects already within ten trials. However, this was not observed when the CubicMouse is operated as the first device (interaction devices* trial* order of devices: $\left.\mathrm{F}_{(1,6)}=4.59, \mathrm{p}=.08\right)$. The interaction thus indicates that the ease of usage of the CubicMouse is indeed inherent in the data, but disappears already after ten trials. 


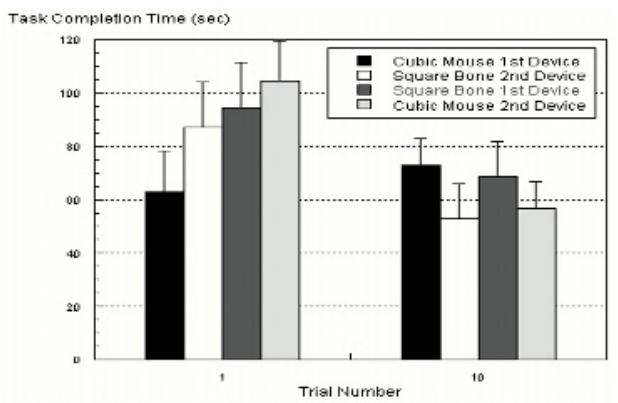

Fig. 5. Mean task completion times (TCTs) and standard errors for the CubicMouse and the SquareBone obtained in trials 1 and 10 separately for the group beginning with the CubicMouse and the one beginning with the SquareBone

Mean usability ratings were with $3.24(\mathrm{se}=.18)$ for the CubicMouse marginally better than for the SquareBone (2.70; se=.16; $\mathrm{F}(1,6)=5.28 ; \mathrm{p}=.06$ ) confirming the slightly better performances with the CubicMouse. Better evaluations of the CubicMouse were also observed in other ratings of task difficulty, of device features, and especially of the difficulty of navigation. Hence, although the CubicMouse requires rather long times for re-grasping since each rod has to be manipulated separately, it seems to have, at least for novice users, several advantages.

\section{General Discussion}

The extended docking task, although very difficult for novice users, provides reliable and valid measures of performance for 12-DOF devices. The results of the experienced users clearly revealed several factors as important for the design of 12DOF devices.

The isotonic CubicMouse was inferior relative to the SquareBone as well as to the YoYo. One important factor for this is certainly the relatively long time it takes to change the grip when manipulating the three separate rods. But, even when subtracting these times, the CubicMouse did not produce docking times as fast as the other two devices. This suggests that some more profound deficit of the CubicMouse leads to its lower performance. One possible factor is that due to the separation of the rods, diagonal movements require so-called "city block" movements which alternate between $\mathrm{x}, \mathrm{y}$, and $\mathrm{z}$ axis control. This requires not only grip changes, but probably also more complex mental processing.

However, the CubicMouse has been preferred by our novice users. Moreover, navigation with the CubicMouse was also regarded by all experienced users as extremely easy. This suggests that an isotonic position control device can serve as a kind of prop for the manipulated object thus facilitating exocentric navigation. But, it cannot be denied that the very intuitive navigation with the CubicMouse led to several impairments due to the fact that it forces the user to maintain a stable body position. Therefore, clutching to disable navigation for precise manipulation of an object should be allowed with the CubicMouse. 
The learning rate did not differ profoundly for all three devices. Although the data of Experiment 3 gave rise to the assumption that the CubicMouse can be more easily controlled by novice users, Experiments 1 and 2 revealed that performance for the CubicMouse is much worse than for the other devices. Our data suggests that the superiority of the CubicMouse disappears already after short usage. In other words, the fact that the learning rates did not differ between devices cannot be interpreted as indication that all devices will produce similar learning rates also for novice users. This has to be studied further within long-term evaluations with novices.

The YoYo and the SquareBone produced comparable performances over five weeks. One important feature differing between the SquareBone and the YoYo is that the former allows for simultaneous control whereas the latter does not. Although simultaneous control might save docking time, different movements with both hands can also lead to interferences. As the data for the SquareBone revealed, lower task completion times go ahead with a higher degree of simultaneous operation. That is, simultaneous control was partly successfully applied by our experienced users arguing for the SquareBone as being principally superior over the YoYo. However, simultaneous navigation and object manipulation occurred only in $2 \%$ of the time. Thus, even if this fraction increases with further expertise, we cannot exclude that the potential saving might be of minor practical importance. To get further estimations about the usefulness of simultaneous control, the conditions under which asymmetric bimanual control can lead to advantages must be explored further $[\underline{2}, \underline{10}, \underline{12}]$.

The comparable performances of the SquareBone and the YoYo in Experiments 1 and 2 suggest that a tactile coordinate frame does not facilitate performance. Nevertheless, as some users objected, our design of the tactile coordinates in the SquareBone can also produce disadvantages. Since the square handles of the SquareBone require to grasp one of the corners in order to move diagonally, the degree to which diagonal movements are possible is reduced. This uncomfortable position of one of the fingers might have produced trajectories avoiding such movements and thus an increase of docking times. Therefore, long-term performance for experts using a Bone with round handles (Fig. 6) would be desirable to assess.

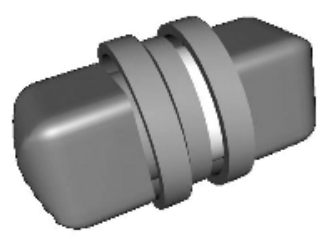

Fig. 6. A new version of the SquareBone: The manipulated handles are round allowing comfortable manipulation of diagonal movements. The square caps on the left and right side provide a tactile coordinate system.

Another fact might argue in favor of the YoYo. Since controlling the YoYo needs time to switch the grip when switching between navigation and object manipulation, one should expect docking times to be about the switching times longer when using the YoYo relative to the SquareBone. But, these additional costs could not be 
observed. Instead, even the fraction of time in which no movement was observed did not differ between both devices. This suggests that switching between navigation and object manipulation produces costs, perhaps due to mental processes, which can hardly be avoided even if a device enables simultaneous performance. The subjective ratings argue also slightly in favor of the YoYo. After all sessions, five of eight participants preferred the YoYo, whereas three favored the SquareBone. Even those who claimed the device was too large leading to accidental movements favored the YoYo suggesting a more fundamental advantage for the YoYo.

\section{Conclusions and Future Work}

This paper presented the results of a first series of user studies for 12-DOF input devices. For the studies, we developed an extended docking task requiring navigation as well as object manipulation. As a main result, we found that for such a complex task, the elastic devices performed significantly better than the isotonic CubicMouse. The newly developed SquareBone is competitive to the YoYo. It allows for simultaneous navigation and manipulation used by very experienced users only. The disadvantage of our current SquareBone lies in prohibiting certain diagonal movements. Nevertheless, with only a few design changes as suggested in Fig 6, the SquareBone holds the potential of becoming an alternative device to the commercially available CubicMouse.

We have investigated the use of these devices for a specific navigation and manipulation task only. Most real world applications additionally require the control of selection and system control tasks besides navigation and manipulation. It is still unclear how well devices can support such interaction modes. There are many more combinations possible to reach 12 or more DOF. The CubicMouse and the Yoyo family are only a starting point for exploring this design space. The combination of isotonic and elastic input bears a lot of potential to support novice and experienced users appropriately, for example by constraining the DOF. This points to reconfigurable smart input devices, which can be adapted or ideally adapt themselves to the task and user.

\section{Acknowledgements}

Thanks to Andreas Simon for providing us with a YoYo, Alexander Kulik for building the SquareBone, David Paneque for keeping the devices in shape, Sonja Gutzeit and Timo Göttel for assisting in data collection, Verena Skuk in data analysis. This work was supported by the VRIB project funded by the German government.

\section{References}

1. Bowman, D., "Interaction Techniques for Common Tasks in Immersive Virtual Environments, Design, Evaluation and Application", PhD-Thesis, Georgia Institute of Technology (1999). 
2. Buxton, W. and Myers, B.A., "A study in two-handed input", Proceedings of CHI 1986 (1986), 321-326.

3. Fitts, P., "The information capacity of the human motor system in controlling the amplitude of movement", Journal of Experimental Psychology, 47 (1954), 381-391.

4. Froehlich, B. and Plate, J., "The CubicMouse: A new device for 3D input", Proceedings ACM CHI 2000 (2000), 526-531.

5. Fröhlich, B., Plate, J., Wind, J., Wesche, G., and Göbel, M.: Cubic-Mouse-Based Interaction in Virtual Environments, IEEE Computer Graphics\&Applications (2000)

6. Gittler, G., "Dreidimensionaler Würfeltest [Three-dimensional cube test]" Weinheim, G, Beltz (1990).

7. Guiard, Y., "Asymmetric division of labor in human skilled bimanual action: The kinematic chain as a model”, Journal of Motor Behavior, 19 (1987), 486-517.

8. Hinckley, K., Pausch, R., Downs, J.H., Proffitt, D., and Kassell, N.F., "The Props-Based Interface for Neurosurgical Visualization", Medicine Meets Virtual Reality, 5th Global Healthcare Grid, Amsterdam, NL, IOS Press (1995), 552-562.

9. Jacob, R., Sibert, L., McFarlane, D., and Mullen, M. 1994.Integrality and separability of input devices. ACM Transactions on Computer-Human Interaction, 1(1), p. 3-26.

10. Leganchuk, A., Zhai, S. and Buxton, W., "Manual and Cognitive Benefits of Two-Handed Input: An Experimental Study", ACM Transactions on Computer-Human Interaction, 5 (1999), 326-359.

11. Masliah M. and Milgram P. Measuring the allocation of control in a 6 degree-of freedom docking experiment. Proceedings of CHI 2000 (2000), 25-32.

12. Mechsner, F., Kerzel, D., Knoblich, G., and Prinz, W., "Perceptual basis of bimanual coordination", Nature, 414 (2001), 69-73.

13. Mine, M., Brooks, F.P., and Sequin, C., "Moving Objects in Space: Exploiting Proprioception in Virtual-Environment Interaction" Proceedings of ACM SIGGRAPH 1997 (1997), 19-26.

14. Pausch, R., Burnette, T., Brockway, D., and Weiblen, M.E., "Navigation and Locomotion in Virtual Worlds via Flight into Hand-Held Miniatures", ACM SIGGRAPH 1995 (1995), 399-400.

15. Simon, A. and Froehlich, B., "The YoYo: A Handheld Device Combining Elastic and Isotonic Input", Interact 2003 (2003).

16. Zhai, S., "Human Performance in Six Degree of Freedom Input Control", PhD-Thesis, University of Toronto (1995). 